\title{
Analytical Nonlinear Response for a Rotor with the Hertz Contact and Clearance
}

\author{
Zijian ZHANG*, Yangyang DONG*, Yanwei HAN** \\ *School of Astronautics, Nanjing University of Aeronautics and Astronautics, 29 Qinhuai Rd, Nanjing, Jiangsu, 210016, \\ China,E-mail: zj.zhang@nuaa.edu.cn \\ **Department of Engineering Mechanics, Henan University of Science and Technology, 263 Kaiyuan Rd, Luoyang, Henan \\ 471023, China, E-mail: yanweihan@haust.edu.cn \\ crossref http://dx.doi.org/10.5755/j01.mech.25.6.24790
}

\section{Introduction}

Rotor system is a kind of mechanical model describing rotating system, which has been applied used in rotating engineering areas $[1,2]$, for example power generation [3], aviation [4], wind turbines [5], turbofan [6], etc. The resonance phenomena in the rotating system $[7,8]$ which will damage rotary machinery, therefore it is necessary to do the work on improving the stability of rotating system [9].

The complexity behavior of nonlinear rotor system is a long-standing problem in the mechanical engineering and rotary machine. So far, many researchers investigate the nonlinear vibration of the nonlinear rotor system with Hertz contact force. Nayak given the theoretical foundation of the observation phenomenon of vibration point contact experiment for the detailed physical explanation [10]. Hess and Soom studied nonlinear vibration with the Hertz contact by using multi-scale perturbation method [11]. Gauthier et al. studied the indentation problem of elastic half space under the action of Coulomb friction and the action of monotonic normal force [12]. Xiao et al. determined the inherent frequency of the undamped vibration of the mass with the Hertzian contact stiffnes by using three methods [13]. Bidi et. al. estimated the contact force and duration of the cylindrical curved double plate by modified Hertz contact theory and shell shear deformation theory [14]. Machado et al. seen a bearing as a polydisperse granular chain which interact Hertzian contact between rolling elements and cage components [15]. Yan, based on the Hertz contact theory and the Winkler foundation, derived analytical formulas of rolling resistance of belt conveyor [16]. Ojolo and Eweina predicted the exact cracking force of cashew nut based on a mathematical model of contact stress of two bodies, the Hertz's theory and assumption of uniaxial compressive load [17]. Nadimi and Fonseca presented the Hertzian response of a single soil grain with the tensile strength by both the experimental and the numerical investigation [18]. Du et al. estimated the tread contact stiffness and given the theoretical support of the wheel face design by using an innovative method based on the Hertz theory [19]. Sladkowski given HertzBeliaev solution of the problem of the contact elastic hemispheres which have been examined by means of FEM [20]. Although various nonlinear rotor models have been proposed in the past hundred years [21-23], an analytical solution of nonlinear rotor with Hertz and clearance has not been achieved [24, 25].
The motivations of this paper were: 1 . To establish a typical rotor system consisting of a disk, a shaft and a stator with Hertz contact; 2. To present the governing equation of complex form for this system by the Lagrangian approach; 3 . To find the natural frequency theoretically for both the free and the forced vibration by the complex harmonic balance (CHBM) method; 4 . To verify the theoretical formula by the numerical integration.

\section{Rotor system}

\subsection{Dynamical model}

As shown in Fig. 1, the rotor model consists of a disc, the stator with Hertz contact and clearance. All the variables of the model can be illustrated in Table 1 .

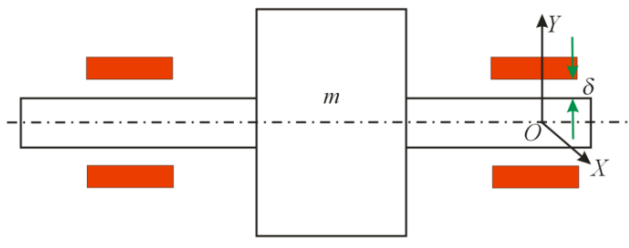

$\mathrm{a}$

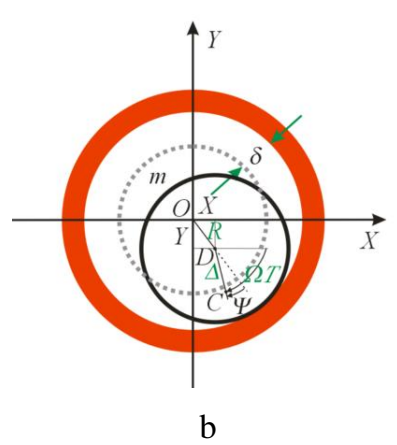

Fig. 1 Model of nonlinear rotor. a-physical model of rotor with the bearing clearance, $b$ - the rotor model with Hertz contact and the clearance

Table 1

Variables of the model

\begin{tabular}{|l|l|}
\hline$k$ & Stator's radial stiffness \\
\hline$\delta$ & Radial clearance of bearing \\
\hline$O$ & Intersection of bearing axis and the plane $X-Y$ \\
\hline$C$ & Mass centre of rotor disc \\
\hline$m$ & Disc's mass \\
\hline$D$ & Geometrical centre of rotor disc \\
\hline$\Delta$ & Disc's eccentricity \\
\hline$\Omega$ & Rotor's spinning speed \\
\hline
\end{tabular}




\subsection{Equation of motion}

Consider the rotor system with Hertz and clearance in Fig. 1, the kinetic energy is:

$$
E=\frac{1}{2} m\left|\frac{d \boldsymbol{Z}}{d T}\right|^{2}=\frac{1}{2} m\left(\left(\frac{d X}{d T}\right)^{2}+\left(\frac{d Y}{d T}\right)^{2}\right),
$$

where: $Z=X+Y i$ is the complex number and $i=\sqrt{-1}$ is the imaginary unit. follow:

The internal energy formula can be obtained as

$$
\begin{aligned}
& U=\frac{2}{5} k H(|\boldsymbol{Z}|-\delta)(|\boldsymbol{Z}|-\delta)^{5 / 2}= \\
& =\frac{2}{5} k H\left(\sqrt{X^{2}+Y^{2}}-\delta\right)\left(\sqrt{X^{2}+Y^{2}}-\delta\right)^{5 / 2} .
\end{aligned}
$$

The Coulomb dissipative formula is:

$$
\Phi=\frac{1}{2} n|\dot{Z}|^{2}=\frac{1}{2} n\left(\dot{X}^{2}+\dot{Y}^{2}\right)
$$

where: $n$ is the viscous damping coefficient.

The external perturbation:

$$
Q=m \Delta \Omega^{2} \exp (i \Omega T)
$$

The Lagrangian $L$ :

$$
L=E-U \text {. }
$$

Using the Lagrangian equation and the Coulomb dissipative function, i.e.:

$$
\frac{\mathrm{d}}{\mathrm{d}}\left(\frac{\partial L}{\partial \dot{\boldsymbol{Z}}}\right)-\frac{\partial L}{\partial \boldsymbol{Z}}+\frac{\partial \Phi}{\partial \dot{\boldsymbol{Z}}}=\boldsymbol{Q}
$$

The differential equation of motion for a nonlinear rotor system with complex clearances was derived:

$$
m \ddot{\boldsymbol{Z}}+n \dot{\boldsymbol{Z}}+k \frac{1}{|\boldsymbol{Z}|} \boldsymbol{Z} H(|\boldsymbol{Z}|-\delta)(|\boldsymbol{Z}|-\delta)^{1.5}=\boldsymbol{Q},
$$

where: $\dot{Z}=d \boldsymbol{Z} / d T=\dot{X}+\dot{Y} i$.

By the transformation of $\omega_{0}^{2}=\frac{k}{m}, \zeta=\frac{n}{2 m \omega_{0}}$, the vector equation for the system (7) can be written in follow form:

$$
\ddot{\boldsymbol{Z}}+2 \zeta \omega_{0} \dot{\boldsymbol{Z}}+\omega_{0}^{2} \frac{1}{|\boldsymbol{Z}|} \boldsymbol{Z} H(|\boldsymbol{Z}|-\delta)(|\boldsymbol{Z}|-\delta)^{1.5}=\Delta \Omega^{2} e^{i \Omega t}
$$

Define the transformation of variables as follows:

$$
z=\frac{Z}{\Delta}, \delta_{0}=\frac{\delta}{\Delta}, t=T \omega_{0}, \omega=\frac{\Omega}{\omega_{0}} .
$$

The rotor system (8) is rewritten as the dimensionless expression as follows:

$$
z^{\prime \prime}+2 \zeta z^{\prime}+z \frac{1}{|z|} H\left(|z|-\delta_{0}\right)\left(|z|-\delta_{0}\right)^{1.5}=\omega^{2} \mathrm{e}^{i \omega t},
$$

where: $z^{\prime}=\frac{\mathrm{d} z}{\mathrm{~d} t}=x^{\prime}+y^{\prime} i$

2.3. Nonlinear restoring forces and potential energy

The expression of dimensionless restoring force is:

$$
\boldsymbol{f}(\boldsymbol{z})=z \frac{1}{|z|} H\left(|z|-\delta_{0}\right)\left(|z|-\delta_{0}\right)^{1.5}=f(x)+f(y) i .
$$

The surfaces of dimensionless forces $f_{x}$ and $f_{y}$ are plotted in Figs. 2, a - c, which are both considered as functions of $x$ and $y$, determined by the real section of formula (11). It is seen from Fig. 2 that $f(x)$ decreases from positive to negative with $x$. From Figs. $2, \mathrm{~b}-\mathrm{d}$, the dimensionless force $f(y)$ is a function of vertical displacement $y$, decided by the imaginary section of bearing clearance $\delta_{0}$ in formula (11). The gradient, especially the stiffness of the mechanism, is determined by the values of $x$ and $y$.
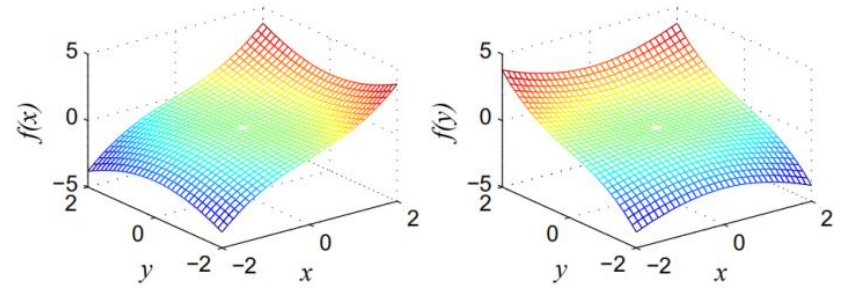

a

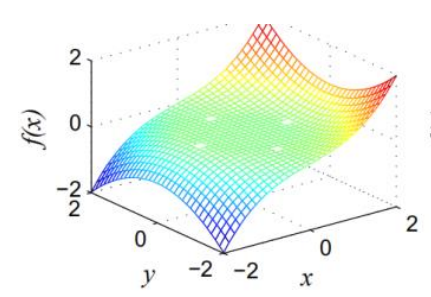

b

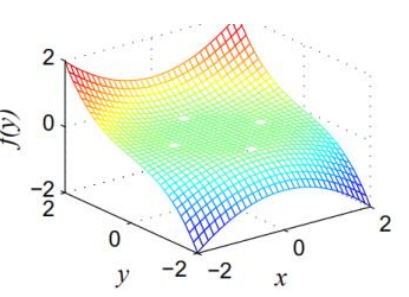

c

d

Fig. 2 The force-displacement curves for different values of stiffness ratio $\alpha$, bearing clearance $\delta_{0}$. a $-\delta_{0}=0.0$, $\mathrm{b}-\delta_{0}=0.0, \mathrm{c}-\delta_{0}=0.5, \mathrm{~d}-\delta_{0}=0.5$

Dimensionless nonlinear potential energy function can be expressed as:

$$
V=\frac{2}{5} H\left(|\mathbf{z}|-\delta_{0}\right)\left(|\mathbf{z}|-\delta_{0}\right)^{2.5}
$$

Fig. 3 shows the nonlinear curves of potential energy $V$ varying with $x$ and $y$, and presents the complex inner surface of the depression. The results show that the potential curves are concave under any combination of parameters. 


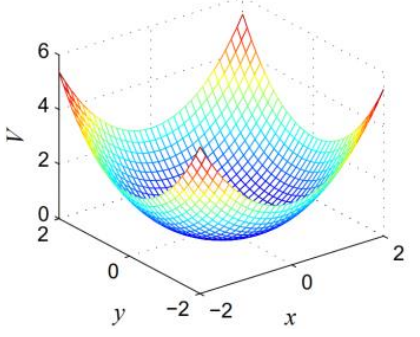

a

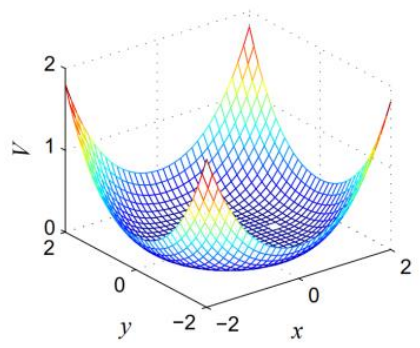

c

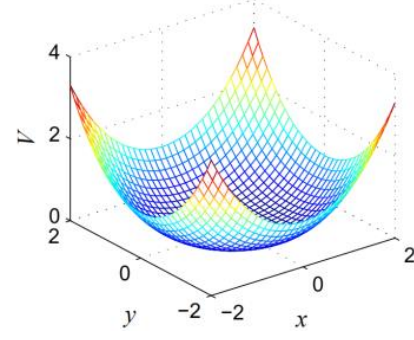

b

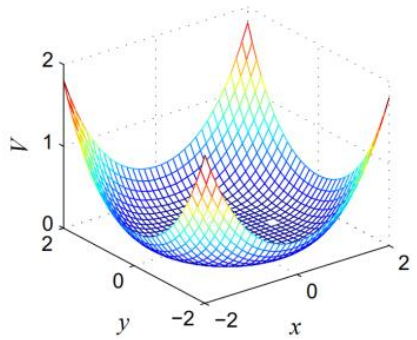

d

$$
T=\frac{2 \pi}{\omega}=\frac{2 \pi}{\sqrt{A^{0.5}}},
$$

here: $\omega$ denotes the natural response frequency and is a function of the response amplitude $A$ of free vibration.

The amplitude and frequency of the rotor are shown in Eq. (17 a). The relationship between amplitude $A$ and frequency $\omega$ are illustrated in Fig. 4. As shown in Fig. 4, a, the amplitude $A$ increases with the frequency $\omega$. In Fig. $4, \mathrm{~b}$ the amplitude $A$ decreases with the periodic $T$.

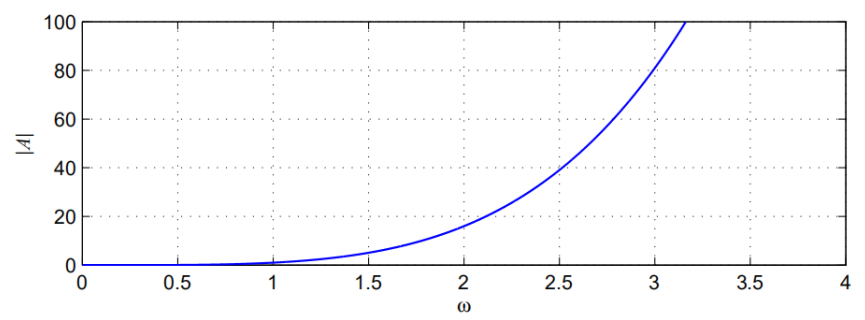

a

Fig. 3 The potential surfaces in the $x-y$ ccoordinate planes for different values of bearing clearance $\delta_{0}$. $\mathrm{a}-\delta_{0}=0.0, \mathrm{~b}-\delta_{0}=0.0, \mathrm{c}-\delta_{0}=0.5, \mathrm{~d}-\delta_{0}=0.5$

The dimensionless nonlinear Hamilton energy function can be expressed:

$$
H=\frac{1}{2}\left|\mathbf{z}^{\prime}\right|^{2}+\frac{2}{5} H\left(|\mathbf{z}|-\delta_{0}\right)\left(|\mathbf{z}|-\delta_{0}\right)^{2.5}
$$

with the help of the Hamilton function Eq. (13), the trajectories could be classified and analyzed.

\section{The free rotation system}

\subsection{Natural frequency}

By letting parameters $\zeta=0, \omega=0, \delta_{0}=0$ the free vibration for the system (10) can be obtained as follows:

$$
z^{\prime \prime}+z|z|^{0.5}=0
$$

It is an autonomous system without gap, damp and external force.

The solution of rotor system (14) of the free vibration can be obtained:

$$
z=A \mathrm{e}^{i \omega t}
$$

And submitting Eq. (15) into Eq. (14), we obtained:

$$
A \mathrm{e}^{i \omega t}\left(-\omega^{2}+A^{0.5}\right)=0
$$

Based on the expression $A \exp (i \omega t) \neq 0$, the natural response frequency $\omega$ in Eq. (14) can be obtained as follows:

$$
\omega= \pm \sqrt{A^{0.5}}
$$

and the vibration period is:

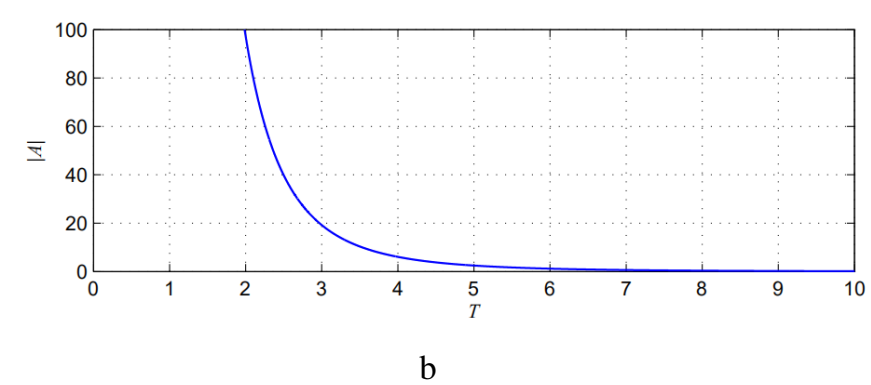

Fig. 4 Natural frequency of free vibration of system. a -the amplitude frequency curve on $(\omega, A)$ plane, $\mathrm{b}$ - amplitude periodic curve on $(T, A)$ plane

\subsection{Hardening stiffness characteristic}

For a nonlinear free rotation system (14), the natural frequencies and periods are physical parameters related to the initial conditions. As shown in Fig. 5, a, the phase diagram of the system (14) at fixed intervals under different initial conditions of $\left(x_{0}, 0,0,0\right)$ with $x_{0}=0,2,4$, 6, 8. As illustrated in Fig. 5, b, the phase portraits of system (14) for the initial conditions of $\left(0,0,0, y_{0}{ }^{\prime}\right)$ with $y_{0}{ }^{\prime}=0,2,4,6,8$. It is found that the higher the amplitude, the longer the corresponding period, so the system behavior has the characteristic of hardening stiffness.

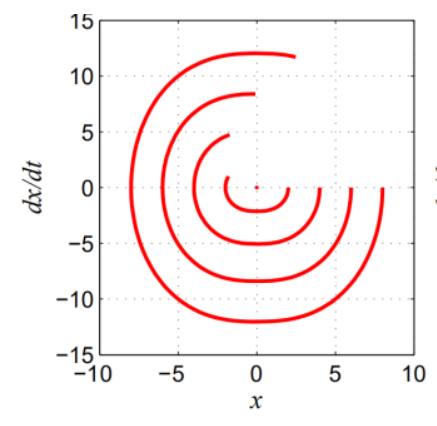

a

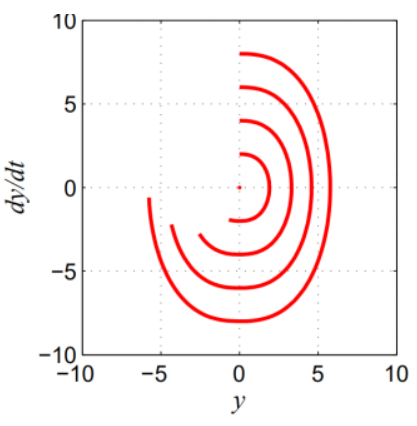

b
Fig. 5 The hardening stiffness characteristic. a - for different initial displacement $\left(x_{0}, 0,0,0\right), \mathrm{b}$ - for different initial velocity $\left(0,0,0, y_{0}{ }^{\prime}\right)$ 


\section{The forced rotating system}

\subsection{Clearance-free forced rotation system}

Under conditions of the viscous damping $\zeta \neq 0, \omega=0, \quad \delta_{0}=0$ and the external periodic force $\omega \neq 0$, the clearance $\delta_{0} \neq 0$, the form of disturbance damped rotor system is as follows:

$$
z^{\prime \prime}+2 \zeta z^{\prime}+z|z|^{1.5}=\omega^{2} \mathrm{e}^{i \omega t},
$$

where: the $\zeta$ is the damping ratio, and the $\omega$ is the external excitation frequency.

\subsubsection{Frequency response characteristics}

The particular solution for the force-rotating system (18) can be defined:

$$
z=A \mathrm{e}^{i(\omega t-\theta)},
$$

submitting Eq. (19) into the force-rotating system (18), then it is can be obtained:

$$
z=H(\omega, A) \Delta,
$$

where: $H(\omega, A)$ is a complex variables function of the frequency $\omega$ and the amplitude $A$. The Amplitude-frequency function is obtained as follows:

$$
H(\omega, A)=\frac{\omega^{2}}{A^{0.5}-\omega^{2}+(2 \zeta \omega) i}=\beta \mathrm{e}^{i \theta},
$$

where the magnification factor $\beta$ for amplitude is:

$$
\beta=\frac{\omega^{2}}{\sqrt{\left(-\omega^{2}+A^{0.5}\right)^{2}+(2 \zeta \omega)^{2}}},
$$

and the phase angle $\theta$ is given as follows:

$$
\theta=\arctan \left(\frac{2 \zeta \omega}{-\omega^{2}+A^{0.5}}\right)
$$

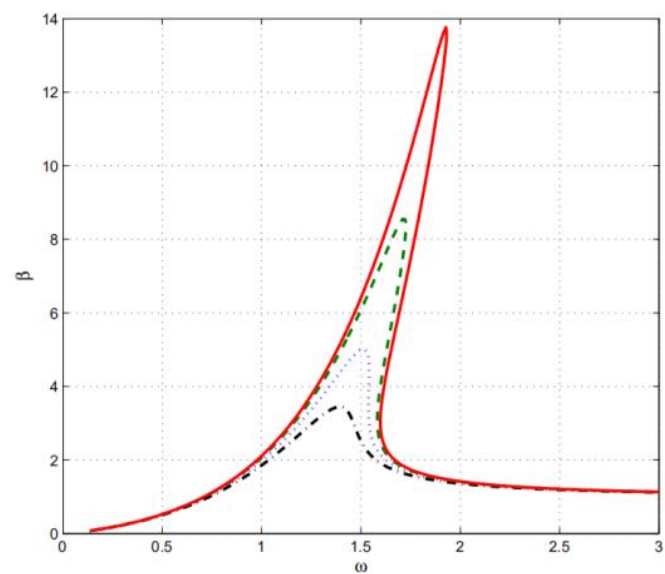

Fig. 6 Amplitude-frequency curves of force-rotating system with different damping ratios $\zeta$. Solid line $\zeta=0.07$, dashed line $\zeta=1.0$, dotted line $\zeta=1.5$, dashed-dotted line $\zeta=2.0$
The relationship of the frequency $\omega$ and the magnification factor $\beta$ is interdependency, named the frequencyamplitude response. From the Fig. 6, the frequency response was plotted by taking the damping ratio $\zeta$ as the control parameter. It was found that there was the jump bifurcation phenomenon.

\subsubsection{Attraction domain of periodic response}

As shown in Fig. 7, we plotted the attracting set in initial plane $\left(x_{0}, y_{0}\right)$ to show attracting regions of the big periodic solution marked by symbol $*$, also the small periodic solution denoted by symbol ${ }^{\circ}$. It was interesting found that the shape of the attraction region looks like a spider.

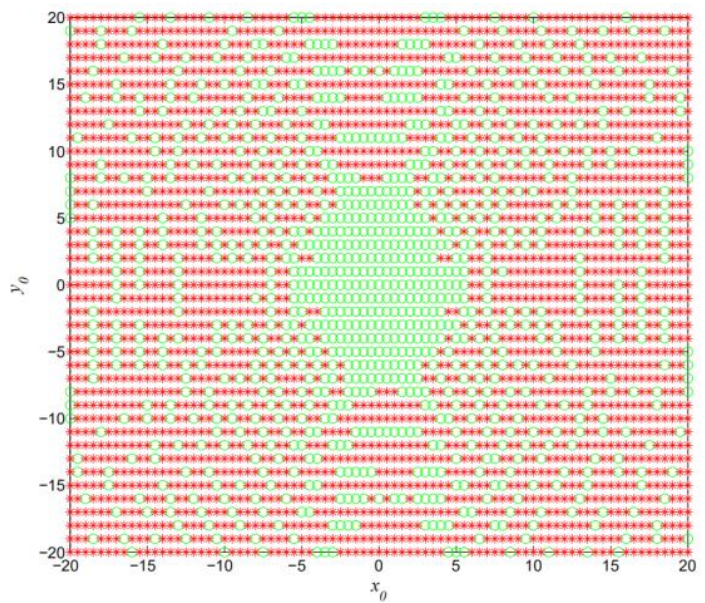

Fig. 7 Attraction domain in $\left(x_{0}, y_{0}\right)$ plane for $\omega=1.7$; $\zeta=0.07$

It is can be seen in Fig. 8, under initial conditions of $*$, a stable large periodic solution is obtained by numerical method in order to show the historical characteristics of time. Figs. 8, a - d give the time histograms of axle trajectory, horizontal and vertical axis trajectory for the large periodic orbit, respectively.
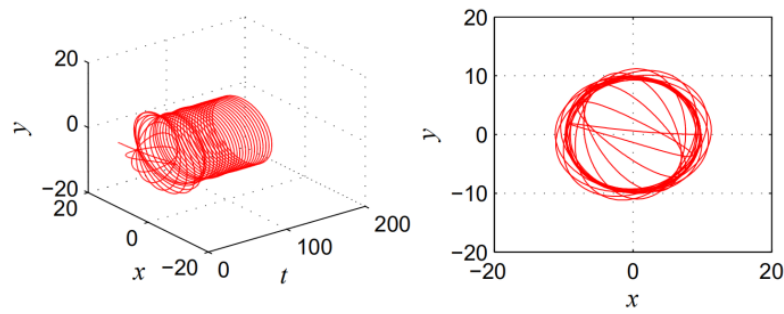

a

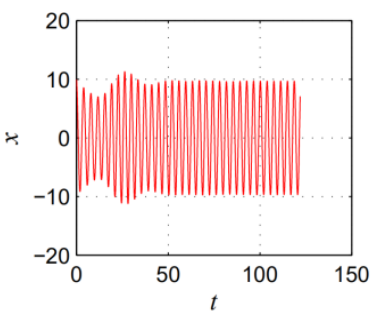

b

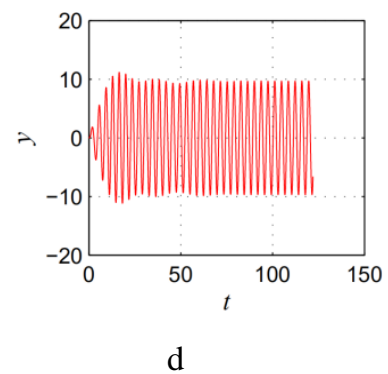

Fig. 8 The big periodic solution for the initial condition $(0$, $0,0,0)$ and $\omega=1.7$. a - three dimensional axial trajectories; b - the plane axial trajectories, c - time history of horizontal direction $x ; \mathrm{d}$ - time history of vertical direction $y$ 
From the Fig. 9, in order to show the historical properties of time, the stable small periodic solutions are given under initial conditions of $\circ$.

However, numerical simulation cannot obtain an unstable periodic orbit, because the unstable orbit is related to the transient motion.

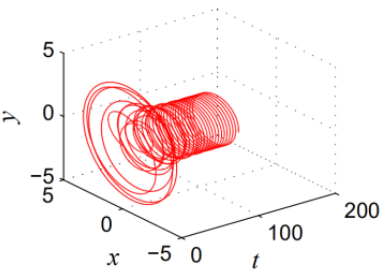

a

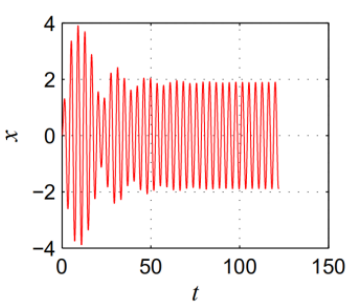

c

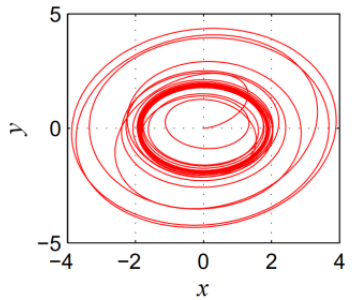

b

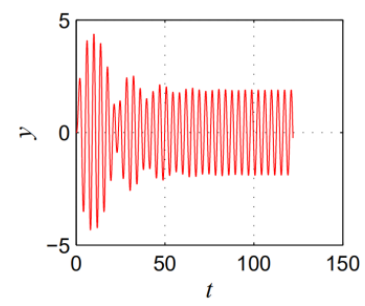

d
Fig. 9 The small periodic solution for the initial condition $(10,0,0,0)$ and $\omega=1.7 . \mathrm{a}-$ three dimensional axial trajectories; $\mathrm{b}$ - the plane axial trajectories, $\mathrm{c}$ - time history of horizontal direction $x$; $\mathrm{d}$ - time history of vertical direction $y$

\subsubsection{General solution}

The general solution of system (18) is assumed as:

$$
z=C_{3} e^{\lambda_{1} t}+C_{4} e^{\lambda_{2} t}+A e^{i(\omega t+\theta)},
$$

where: $\lambda_{1}=\omega_{0}, \lambda_{2}=-\omega_{0}$ the first two terms are general regular solutions, and the last one is special solutions.

Using the initial conditions $\left(x_{0}, x_{0}^{\prime}, y_{0}, y_{0}^{\prime}\right)$ we obtained the:

$$
\begin{aligned}
& z_{0}=C_{3}+C_{4}+A e^{i \theta}, \\
& z_{0}^{\prime}=\lambda_{1} C_{3}+\lambda_{2} C_{4}+i A \omega e^{i \theta},
\end{aligned}
$$

here: $C_{3}, C_{4}$ denote undetermined parameters. (25), we get:

By establishing synchronization in the equation

$$
\begin{aligned}
& C_{3}=\frac{r_{0} \lambda_{2}-\dot{r}_{0}-A e^{i \theta} \lambda_{2}+A \omega e^{i \theta} i}{\lambda_{2}-\lambda_{1}}, \\
& C_{4}=\frac{\lambda_{1} r_{0}-\dot{r}_{0}-A e^{i \theta} \lambda_{1}+A \omega e^{i \theta} i}{\lambda_{1}-\lambda_{2}} .
\end{aligned}
$$

Substituting coefficients (26) and (27) into the Eq. (24), the general solution of system (18) was obtained as:

$$
z=\frac{r_{0} \lambda_{2}-\dot{r}_{0}-A e^{i \theta} \lambda_{2}+A \omega e^{i \theta} i}{\lambda_{2}-\lambda_{1}} e^{\lambda_{1} t}+
$$

$$
\begin{aligned}
& +\frac{\lambda_{1} r_{0}-\dot{r}_{0}-A e^{i \theta} \lambda_{1}+A \omega e^{i \theta} i}{\lambda_{1}-\lambda_{2}} e^{\lambda_{2} t}+ \\
& +A e^{i(\omega t+\theta)},
\end{aligned}
$$

where: $\lambda_{1}=-\zeta+\sqrt{\zeta^{2}-1}, \lambda_{2}=-\zeta-\sqrt{\zeta^{2}-1}$,

$$
A=\frac{\omega^{2}}{\sqrt{\left(-\omega^{2}+A^{0.5}\right)^{2}+(2 \zeta \omega)^{2}}}, \theta=\arctan \left(\frac{2 \zeta \omega}{-\omega^{2}+\varepsilon A^{0.5}}\right) \text {. }
$$

4.2. The forced rotation system with clearance

\subsubsection{Amplitude frequency}

The force-rotating model for system (8) with the rotor system Hertz and clearance can be rewritten in the following form:

$$
z^{\prime \prime}+2 \zeta z^{\prime}+z H\left(|z|-\delta_{0}\right)\left(|z|-\delta_{0}\right)^{1.5}=\omega^{2} \mathrm{e}^{i \omega t} .
$$

The above system is an inherent nonlinear system with jump function, signum function, and 1.5 order fractal nonlinearity.
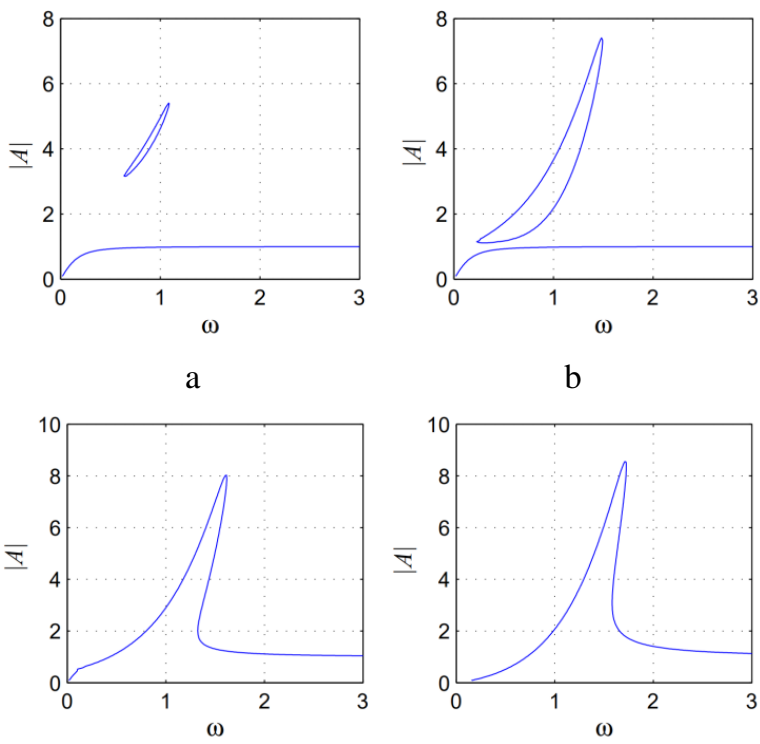

b

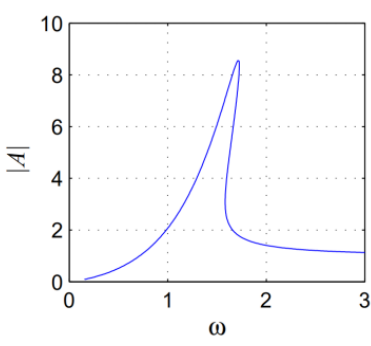

c

d

Fig. 10 The amplitude frequency curves for the nonlinear rotor system with clearance $\delta$. a - for $\delta=2, \mathrm{~b}-$ for $\delta=1, \mathrm{c}-$ for $\delta=0.5, \mathrm{~d}-$ for $\delta=0.0$

For rotating system (29) with clearances, the derivation of the amplitude-frequency relationship is as follows.

$$
A=\frac{\omega^{2}}{\sqrt{\left(-\omega^{2}+\frac{1}{A} H(A-\delta)(A-\delta)^{1.5}\right)^{2}+(2 \zeta \omega)^{2}}} .
$$

pressed:

The phase-frequency relationship $\theta$ was ex-

$$
\theta=\arctan \left(\frac{2 \zeta \omega}{-\omega^{2}+\frac{H(A-\delta)(A-\delta)^{1.5}}{A^{2}}}\right) .
$$


Fig. 10 shows the effect of clearance $\delta=2,1,0.5$ and 0.0 on the amplitude-frequency resonance curve of a forced rotating rotor under Hertz contact force and clearance. It is found that the system behaviors hysteretic characteristic. It should be emphasized that the amplitude $A$ can be reduced and shifted to the left by increasing the clearance coefficient $\delta$ of the main resonance region. Therefore, there is a method to reduce the risk of rotor rotation, that is, to increase clearance $\delta$.

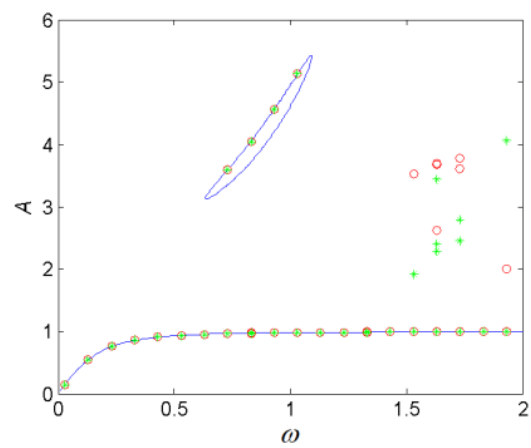

a

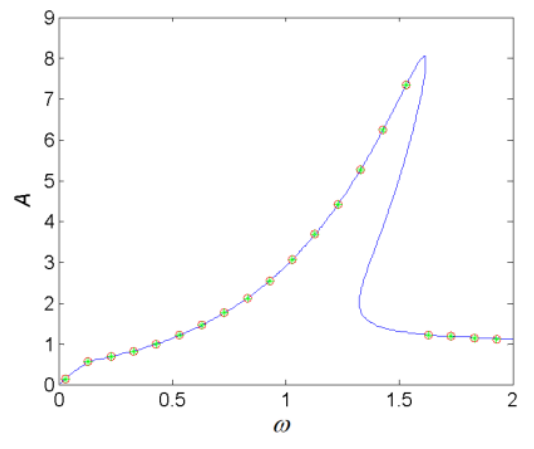

c

\subsubsection{Numerical simulation}

The Runge-Kutta method is used to simulate the amplitude-frequency response in steady state, and the reliability of the theoretical response formula of perturbed rotor system is verified.

As shown in Fig. 11, the numerical simulation results (expressed in circles ${ }^{\circ}$ ) verify the accuracy of theoretical results (expressed in solid line -) for the presented rotor system. Several comparisons between theoretical results and numerical results show that periodic dynamics has a good correlation.

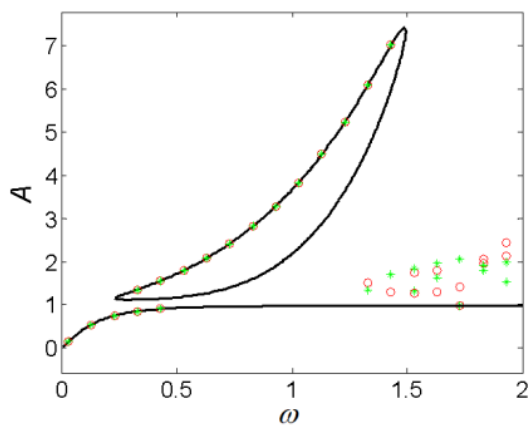

b

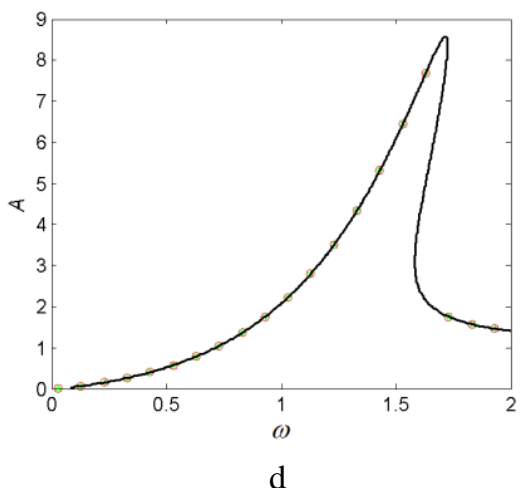

d

Fig. 11 The comparison of theoretical results and numerical simulation: $\mathrm{a}-$ for $\delta=2, \mathrm{~b}-$ for $\delta=1, \mathrm{c}-$ for $\delta=0.5, \mathrm{~d}-$ for $\delta=0.0$

\section{Conclusions}

In this paper, the frequency response of a rotor system with piecewise nonlinearity of Hertzian contact fractal power and bearing clearance is studied. A complex equation of motion for this rotor system was deduced using the Lagrange equation. The natural frequency and phase portraits of the unperturbed rotor system were obtained to demonstrate hardening stiffness characteristics. The amplitude frequency responses and attraction basins of multiple solutions for the perturbed rotatory system without clearance were investigated. The analytical form of amplitude frequency responses of the perturbed system with clearance were analyzed. The theoretical results are verified by numerical simulation and in good agreement with the numerical results.

\section{Acknowledgements}

The authors acknowledge the financial supports from the Natural Science Foundation of China (Grant No. 51705241, No. 11802118), the National Science Foundation of Jiangsu Province (Grant No. BK20170808).

\section{References}

1. Dimarogonas, A.; Paipetis, S. A.; Chondros, T. G. 2013. Analytical Methods in Rotor Dynamics, 2nd edition, Springer-Verlag: New York.

2. Bouzidane, A.; Thomas, M. 2008. An electrorheological hydrostatic journal bearing for controlling rotor vibration, Computers \& Structures 86(3-5): 463 472.

https://doi.org/10.1016/j.compstruc.2007.02.006.

3. Gustavsson, R. K.; Aidanpaa, J. O. 2006. The influence of nonlinear magnetic pull on hydropower generator rotors, Journal of Sound and Vibration 297 (3): 551-562. https://doi.org/10.1016/j.jsv.2006.04.016.

4. Yu, P.; Zhang, D.; Ma, Y.; \& Hong, J. 2018. Dynamic modeling and vibration characteristics analysis of the aero-engine dual-rotor system with fan blade out, Mechanical Systems and Signal Processing 106: 158-175. https://doi.org/10.1016/j.ymssp.2017.12.012. 
5. Ishida, Y.; Yamamoto, T. 2002. Linear and Nonlinear Rotordynamics: A Modern Treatment with Applications, 2nd edition, Wiley-VCH: Weinheim.

6. Muszynska, A. 1989. Rotor-to-stationary element rubbing-related vibration phenomena in rotating machinery-literature survey, Shock and Vibration Digest 21(3): $3-11$. https://doi.org/10.1177/058310248902100303.

7. Jeffcott, H. H. 1919. The lateral vibration of loaded shafts in the neighbourhood of a whirling speed - the effect of want of balance, Philosophical Magazine 37 (219): 304-314. https://doi.org/10.1080/14786440308635889.

8. Rao, J. S. 2011. History of Rotating Machinery Dynamics, Springer-Verlag: Netherlands

9. Kramer, E. 1993. Dynamics of Rotors and Foundations, Springer-Verlag: Berlin Heidelberg.

10. Nayak, P. R. 1972. Contact vibrations, Journal of Sound and Vibration 22(3): 297-322. https://doi.org/10.1016/0022-460X(72)90168-X.

11. Hess, D. P.; Soom, A. A. 1991. Normal vibrations and friction under harmonic loads: part I-Hertzian contacts. Journal of Tribology-ASME 113(1): 80-86. http://dx.doi.org/doi:10.1115/1.2920607.

12. Gauthier, A.; Knight, P. A.; Mckee S. 2007. The Hertz contact problem, coupled Volterra integral equations and a linear complementarity problem, Journal of Computational and Applied Mathematics 206(1): 322 340. http://dx.doi.org/doi:10.1016/j.cam.2006.07.008.

13. Xiao, H.; Brennan, M. J.; Shao, Y. 2011. On the undamped free vibration of a mass interacting with a Hertzian contact stiffness. Mechanics Research Communications 38(8):560-564. https://doi.org/10.1016/j.mechrescom.2011.07.012

14. Bidi, A.; Liaghat, G.; Rahimi, G. 2018. Low-velocity impact on cylindrically curved bilayers. Proceedings of the Institution of Mechanical Engineers, Part K: Journal of Multi-body Dynamics 232(4): 568-576. https://doi.org/10.1177/1464419318756661.

15. Machado, C.; Baudon, S.; Guessasma, M.; Bourny, V.; Fortin, J.; Bouzerar, R.; Maier, P. 2017. An original DEM bearing model with electromechanical coupling. International Journal of Computational Methods 16: 1840006.

16. Yan, L. 2018. Investigation on indentation rolling resistance of belt conveyor based on Hertz contact theory compared with one-dimensional Winkler foundation. Advances in Mechanical Engineering 10(7): 1-9. https://doi.org/10.1177/1687814018783938.

17. Ojolo, J. S.; Eweina, B. A. 2019. Predicting cashew nut cracking using hertz theory of contact stress. Journal of the Saudi Society of Agricultural Sciences 18(2): 157-167.https://doi.org/10.1016/j.jssas.2017.04.002.

18. Nadimi, S.; Fonseca, J. 2017. On the tensile strength of soil grains in Hertzian response [J]. EPJ Web of Conferences 140: 07001. https://doi.org/10.1051/epjconf/201714007001.

19. Du, Z.; Zhou J. C.; Wen, X. X. 2019. Research of normal contact stiffness of straddle-type monorail tyres based on fractal theory, Mechanika 25(3): 248-254. https://doi.org/10.5755/j01.mech.25.3.22265.
20. Sladkowski, A. 2005, Accuracy analysis of the solution of spatial contact problem by means of the FEM, Mechanika 3(53): 17-21.

https://doi.org/10.5755/j01.mech.53.3.13047.

21. Shen, J. F.; Xu, S.; Liu W. W.; Yang J. J. 2017. Fractal model of normal contact stiffness between two Spheres of joint interfaces with simulation, Mechanika 23(5): 703-713. http://dx.doi.org/10.5755/j01.mech.23.5.19356.

22. An, B.; Ma, D.; Wang, P.; Zhou, J.; Chen, R., Xu, J.; Cui, D. 2019. Assessing the fast non-Hertzian methods based on the simulation of wheel-rail rolling contact and wear distribution. Proceedings of the Institution of Mechanical Engineers, Part F: Journal of Rail and Rapid Transit 0(0): 1-14.

https://doi.org/10.1177/0954409719848592.

23. Argatov, I.; Kachanov, M.; Mishuris, G. 2017. On the concept of "far points" in Hertz contact problems. International Journal of Engineering Science 113(2017): 20-36. https://doi.org/10.1016/j.ijengsci.2016.11.009.

24. Zhang, J.; Lu, X.; Lin J.; Ma, L.; Wang, J. 2017. Dynamic analysis of a rotor-bearing-sfd system with the bearing inner race defect, Shock and Vibration 2017: 1-13. https://doi.org/10.1155/2017/2489376.

25. Zdancevicius, E.; Kacianauskas, R.; Zabulionis, D. 2017. Improvement of viscoelastic damping for the hertz contact of particles due to impact velocity, Procedia Engineering 172: 1286-1290.

https://doi.org/10.1016/j.proeng.2017.02.156.

\section{Z. Zhang, Y. Dong, Y. Han}

\section{ANALYTICAL RESPONSE FOR A NONLINEAR ROTOR WITH THE HERTZ BEARING AND CLEARANCE}

S u m m a r y

This paper focuses on a nonlinear frequency response of a rotor system with power of Hertz contact and piecewise nonlinearities of bearing clearance. A complex equation of motion for this rotor system was deduced using the Lagrange equation. The natural frequency and phase portraits of the unperturbed rotor system were obtained to demonstrate hardening stiffness characteristics. The amplitude frequency and attraction basins of multiple solutions for the perturbed rotatory system without clearance were investigated. The analytical forms of amplitude frequency of the perturbed system with clearance were analyzed. Through numerical simulation, the correctness of the theoretical formula is verified, and it is in good agreement with the numerical results.

Keywords: nonlinear rotor, Hertiz contact force, clearance, hysteretic bifurcation.

Received September 19, 2018 Accepted November 21, 2019 